\title{
Ensayo mecánico de la rotura fisaria. Estudio esperimental en el férmur del cordero
}

\author{
FORRIOL, F. Y CAÑADELL, J. \\ Laboratorio de Ortopedia Experimental. Departamento de Cirugía Ortopédica y Traumatología. \\ Clinica Universitaria. Facultat de Medicina. Universidad de Navarra
}

\section{RESUMEN}

La distracción fisaria es una técnica empleada en Cirugía Ortopédica, que consiste en separar, con un fijador externo, la epífisis de la metáfisis de los huesos largos, generalmente a nivel distal del fémur o proximal de la tibia, para efectuar elongaciones o correcciones angulares.

En el presente trabajo se efectúan roturas en 36 fémures de corderos, con una máquina de ensayos universal, analizando la fuerza y el desplazamiento necesarios para producir la rotura fisaria. No hemos encontrado diferencias significativas entre los fémures de machos y de las hembras y se demuestra la importancia que tienen las inserciones capsulares en este mecanismo.

Palabra clave: Distribución fisaria - Fijador externo Cartílago de crecimiento - Epifisiolisis

\section{SUMMARY}

The fissary distraction is a technique used in Orthopedic Surgery, consisting in separating, with an external fixer, the epiphysis from the metaphysis of the long bones, generally in a distal level of the fermur or proximal to the shin-bone, in order to do elongations or angular corrections.

In the present study 36 lamb femurs have been fractured, with a universal testing machine, analyzing the strength and the necessary displacement to produce the fissary fracture. We have not found significant differences between the male and female femurs and it has been demonstrated the importance that the capsular insertions have in this method.

Key words: Fissary distraction - External fixer Growth cartilage - Epiphysiolasis.

\section{INTRODUCCIÓN}

La distracción fisaria es una técnica de frecuente utilidad en Cirugía Ortopédica que permite mediante la separaciónde la epífisis elongar los huesos, corrigiendo, si fuera preciso, también las desviaciones axiales. $1,4,5,6,8,9,9,16,17,18,22-24,33,36,37,40,41$

Sin embargo, ha existido, sobre su mecanismo de acción una gran controversia en la literatura entre los defensores de la condrodiastasis ${ }^{1,8,9}$, sostienen que al efectuar una tracción longitudinal en un hueso en crecimiento, colocando unos clavos a nivel diafisario y otros a nivel epifissario, se produce un estiramiento del cartíla- go, y otros que mantienen la separación real entre la metáfisis y la epífisis dejando un espacio que contienen un hematoma que osifica posteriormente como si se tratase de una fractura. $4,5,10,11,22-24,26$

El objetivo del presente estudio es analizar las curvas fuerza/desplazamiento que se producen al separar la epífisis distal del fémur del resto del hueso en el cordero en crecimiento.

\section{MATERIAL Y MÉTODO}

Se utilizaron 36 fémures de corderos con edades comprendidas entre 2 y 8 meses todos ellos con cartílago de crecimiento activo comprobado mediante radiografía y visualización macroscópica.

Para la realización del presente trabajo se utilizó una máquina de ensayos universal (Instron 4502), con unas mordazas especiales que semejan a la barra de un fijador externo con orificios para la introducción de clavos de $5 \mathrm{~mm}$ de diámetro (Apex).

Después de pelar los huesos se colocaron, lateralmente, dos clavos proximales en la diáfisis femoral y dos clavos distales, perpendiculares a los anteriores, en la epífisis distal del hueso. Introduciendo los clavos en las mordazas se procedió al ensayo a tensión, con una velocidad de $10 \mathrm{~mm} / \mathrm{min}$ finalizando el mismo cuando se produjo la separación entre la epífisis y la metáfisis femoral.

Dividimos los huesos según el sexo de los animales dejando un tercer grupo de animales de sexo desconocido.

En un grupo de 7 fémures se conservó la capsula articular adherida al hueso para estudiar la influencia de la inserción de las partes blandas.

Se analizaron las gráficas fuerza/desplazamiento en conjunto, en los machos, en las hembras y también en los huesos que conservaron sus inserciones capsulares.

\section{RESULTADOS}

Todos los huesos rompieron a nivel fisario produciéndose una perfecta separación entre la metáfisis y la epífisis, observando su morfología, cuatro mamelones epifisarios que se incrustan en la metáfisis.

No se observaron diferencias entre las fuerzas a la rotura y los desplazamientos efectuados en las hembras y en los machos (tabla 1). 
TABLA 1

\begin{tabular}{|c|c|c|c|c|c|}
\hline & $x$ & $D S$ & $E S$ & Minimo & Máximo \\
\hline \multicolumn{6}{|l|}{ GLOBAL } \\
\hline Desplazamiento (mm) & 4.73 & 2.39 & 0.41 & 1.43 & 9.39 \\
\hline Fuerza $(\mathrm{Kg})$ & 46.42 & 16.21 & 2.78 & 13.82 & 84.79 \\
\hline \multicolumn{6}{|l|}{ HEMBRAS } \\
\hline Deplazamiento (mm) & 4.89 & 3.00 & 0.9 & 1.43 & 9.39 \\
\hline Fuerza $(\mathrm{Kg})$ & 46.51 & 19.36 & 5.83 & 13.82 & 84.79 \\
\hline \multicolumn{6}{|l|}{ MACHOS } \\
\hline Desplazamiento (mm) & 4.35 & 1.94 & 0.58 & 1.68 & 7.57 \\
\hline Fuerza $(\mathrm{Kg})$ & 46.68 & 15.32 & 4.62 & 25.19 & 69.92 \\
\hline
\end{tabular}

Analizando las gráficas fuerza/desplazamiento de los 36 fémures en conjunto (fig. 1) así como de las gráficas correspondientes a los machos (fig. 2) y de las hembras (fig. 3) se observó como hasta conseguir una epifisiolisis durante una distracción fisaria se sigue un comportamiento lineal.

La gráfica de la distracción fisaria efectuada en huesos que conservan las inserciones capsulares se observa en todos ellos un comportamiento lineal hasta que se obtiene la epifisiolisis, después disminuían las fuerzas y volvían a subir hasta que se rompian las inserciones capsulares. Este comportamiento, con valores de mayor o menor magnitud, se constató en todos los fémures analizados.

\section{DISCUSIÓN}

El comportamiento del cartílago de crecimiento ante diferentes solicitaciones mecánicas fué muy discutido durante el pasado siglo XIX. Diferentes autores propusieron que la comprensión o la tensión potenciaban o retardaban el crecimiento condral.

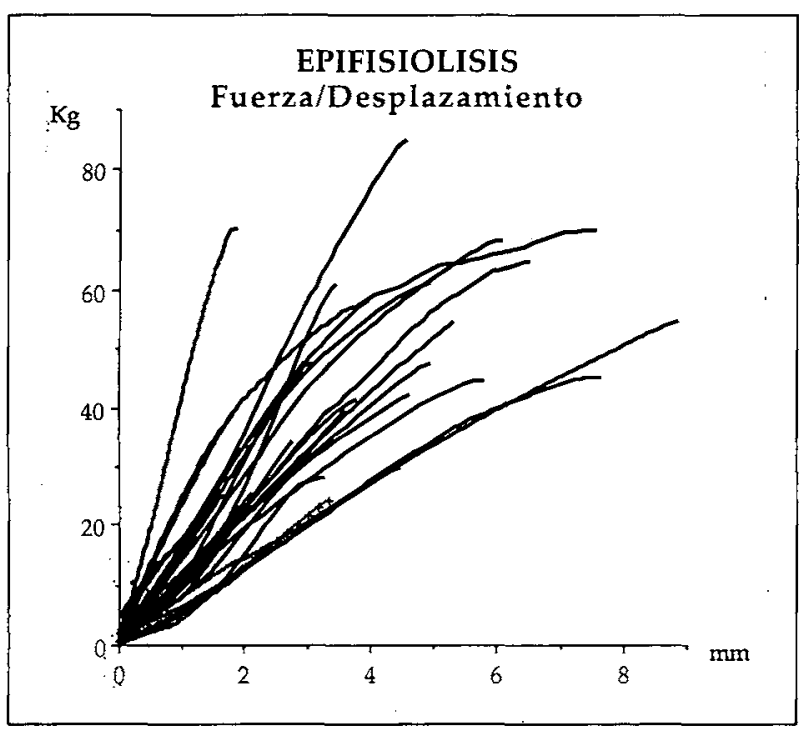

Figura 1.

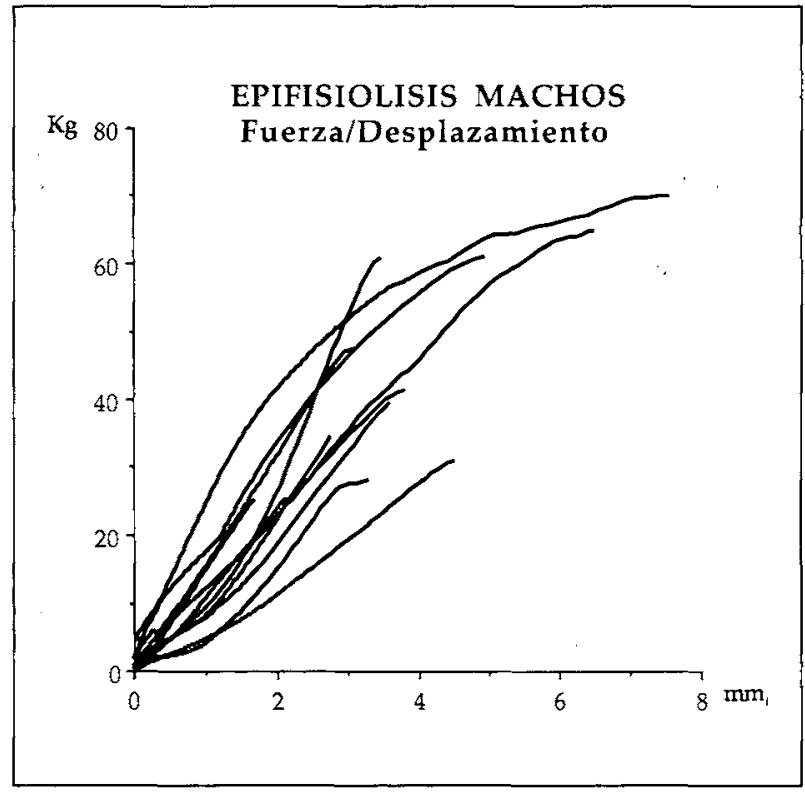

Figura 2.

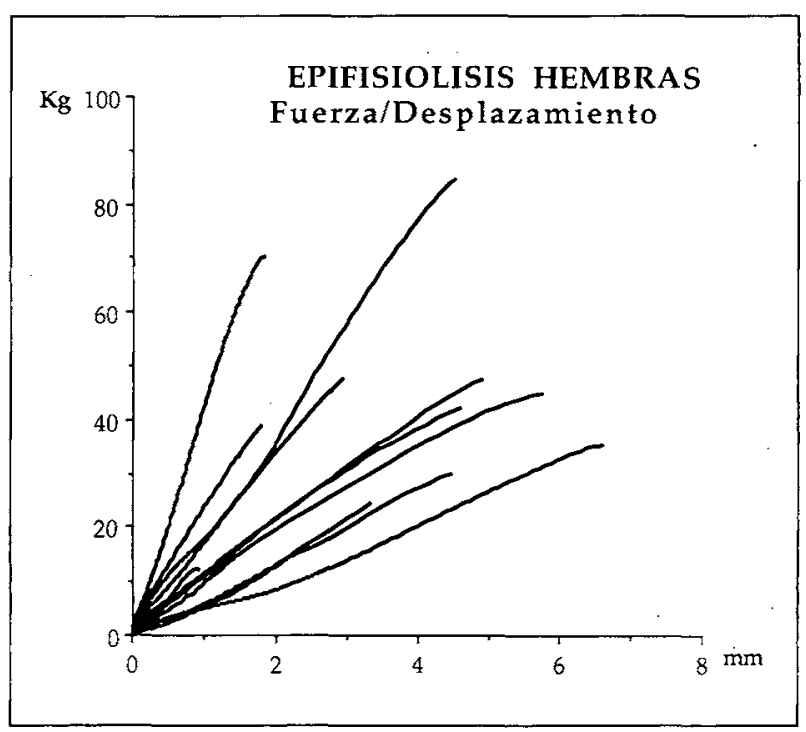

Figura 3. 
Mecánicamente el cartílago humano se comporta siguiendo el denominado modelo bifásico KLM (KueiLai-Mow $)^{25}$ que tiene presentes aspectos dependientes del tiempo en la curva carga/deformación en función la resistencia al transporte de líquido intersticial dado por la permeabilidad de la fase sólida del cartílago (colágeno y proteoglicanos), asociados ambos a las solicitaciones mecánicas y con los efectos tanto iónicos como electroestáticos.

Hert, 1964, concluyó que las fuerzas mecánicas ejercidas por tracción del periostio y el pericondrio en dirección longitudinal, la tensión de los músculos y ligamentos y la carga estática juegan un papel importante en la regulación local de la actividad de crecimiento. Generalmente el tejido cartilagionoso y óseo están sometidos a la acción muscular que se insertan en el periostio de la diáfisis y de la metáfisis. Durante el crecimiento estas fuerzas son capaces de modificar el patrón de crecimiento. Los cambios de tensión en diferentes áreas del periostio puede ser un factor que conlleve un aumento de crecimiento en ciertas porciones de la fisis ${ }^{19}$.

En los niños son frecuentes las lesiones del cartilago de crecimiento. Son raras las lesiones por tracción, los accidentes suelen producirse por solicitaciones de deslizamiento. Para prevenirlo la fisis suelen presentar ondulaciones lo que evita que estas solicitaciones actuen de forma uniforme y al mismo tiempo sobre la totalidad de la fisis. En algunas ocasiones, las placas epifisarias de muchos huesos forman un ángulo tan grande como sea posible con los planos de máximo cizallamiento.

El mecanismo directo no suele producir un desprendimiento epifisario pues en la mayoría de los casos se producen por un mecanismo indirecto, un varo o valgo forzados combinados a una hiperextensión. Las inserciones musculares y ligamentosas juegan en este mecanismo un papel muy importante.

Pietravissa, 1957, estudió la patogenia de los desprendimientos epifisarios en animales, bajo anestesia, y en cadáveres, utilizando huesos largos desprovistos de musculatura, sometidos a tracción axial, en varo, valgo, hiperextensión e hiperflexión articular y a tracción asociada con movimientos anormales. Harris, 1950, por su parte, desarrolló un sencillo modelo experimental para producir desprendimientos epifisarios tibiales atravesando la epífisis con una aguja unida a un cubo donde dejaba caer, poco a poco, arena para ver cuando se produce la rotura. Bright y Elmore, 1968, lo repiten en ratas, mientras que Pelker y Moen, 1983, produjeron roturas de cartílago de crecimiento mediante transfixiones a ambos lados de la fisis aplicando una fuerza de tracción axial o con un componente de rotación, estudiando la línea de rotura y relacionando con la fuerza de aplicación.

En su trabajo experimental con conejos, Oliete, 1984 llega a la conclusión de que la edad, el sexo del animal, la localización de la epífisis y el punto donde se aplica la fuerza son el factor acondicionante más importante y demuestra que los cartílagos más frágiles son los que tienen la arquitectura fisaria más sencilla.

Actualmente el análisis de la distracción fisaria ha despertado de nuevo el interés por conocer la respuesta mecánica, histológica y bioquímica del cartílago de crecimiento. El área próxima a la físis es, durante el crecimiento, una zona ideal para aplicar métodos de elongación por sus especiales características para conseguir la curación ósea. $3,16,17,18,20,33,40,41$ La tracción del cartílago de crecimiento produce una fractura que permite elongar el hueso en un osteotomías óseas consiguiendo una rápida consolidación. En nuestro estudio se analiza la relación de la fuerza y las elongaciones necesarias del fijador externo en relación con el sexo y también el papel que juegan las inserciones capsulares y ligamentosas sobre las fuerzas de distracción que como ya señalase Pietravissa, 1957, son capaces de transformar las fuerzas aplicadas. Al comienzo de la distracción fisaria, el tejido cartilaginoso sufre una deformación pero para lograr una elongación es imprescindible que a metáfisis se separe de la epífisis, produciéndose una epifisiolisis ${ }^{17,29}$ con la consiguiente neoformación ósea en el área de elongación ${ }^{16,22-24}$.

Durante este proceso, el periostio se rompe a nivel de la inserción del anillo pericondral produciendo un hematoma que rellena la separación ${ }^{12,18}$, que posteriormente será reemplazado por tejido fibroso. ${ }^{22-24}$

Fjeld y cols., 1988, demuestran que con una distracción diaria del $0.25 \%$ de la longitud ósea se producen separaciones epifisarias en todos los animales entre el día 5 y 9 . En todos los estudios experimentales de distracción fisaria sea ha observado una separación del cartílado de crecimiento de la metáfisis ${ }^{7,10,11,17,31,33,37}$ y el aumento de la longitud del heuso se consigue con la separación entre la metáfisis y la epífisis ${ }^{26}$. El patrón característico de estrías en la zona de elongación, visible radiologicamente, observándose, ocasionalmente, una zona central radiolúcida en el área de elongación ${ }^{24}$.

También De Pablos y cols., 1986, 1990, demostraron la producción constante de fracturas metafiso-epifisarias, independiente de la velocidad de distracción diaria, estableciendo una relación entre las variaciones morfológicas del cartílago de crecimiento y la velocidad de elongación utilizada. En los fémures de oveja con una velocidad de elongación de $0.5 \mathrm{~mm} / \mathrm{dia}$, el cartílago permanece normal en todas las fases. Sin embargo, en fémures elongados $1 \mathrm{~mm} /$ dia y especialmente, 2 $\mathrm{mm} /$ dia, se observaron lesiones evidentes a los 45 dias y 4 meses después de la elongación. En un estudio experimental realizado en corderos $^{21}$ se demuestra que la distracción fisaria puede realizarse a cualquier edad previa al cierre fisario siempre que la epifisiolisis sea progresiva y la distracción lleve un ritmo inferior a 6.4 veces la tasa de crecimiento del hueso a alargar.

Sin embargo, una fuerza de distracción gradual de pequeña intensidad que actúe simetricamente sobre la placa de crecimiento puede, según algunos autores, $1,2,8,9$ conseguir un crecimiento óseo sin llegar a producir una epifisiolisis constituyendo lo que se ha denominado condrodiastasis. En esta línea, Sledge y Noble, 1978, demuestran la estimulación del cartílago de crecimiento aplicando fuerzas de estimulación distracción de 1-2 Kg sobre la fisis, con un incremento de la división celular y un aumento de la síntesis de polisacáridos sulfatados. Tercedor y cols., 1988, observan a largo plazo la disminución de crecimientode la tibia alargada. Hay una reacción hiperplásica inicial en todas las capas celulares del cartílago de crecimiento y una atrofía posterior. 
Durante la rotura del cartílago epifisario las fuerzas de distracción alcanzan 0-150 $\mathrm{kp}^{22}$ o $28-58 \mathrm{~kg}^{7}$.

No hemos encontrado diferencias en la distracción fisaria entre los fémures de ambos sexos. Tampoco Skak y cols., 1987, encuentran diferencias sexuales en su revisión de las epifisiolisis que por otra parte se considera, clásicamente, más frecuente en el sexo masculino que en el femenino, en una proporción aproximada de 6:1, lo qeu se explica por la mayor agresividad de los niños en sus juegos aunque la fuerza necesaria para producir una epifisiolisis aumenta considerablemente con la administración de estrógenos ${ }^{14}$. Diferentes trabajos corraboran esta afirmación, en tibias de rata la resistencia del cartílago de crecimiento es más grande en hembras que en machos de la misma edad ${ }^{2}$ y Oliete, 1984, también concluye que las hembras de conejos, ofrecen mayor resistencia y ofrecen menor frecuencia de epifisiolisis, siendo esta más sencilla a nivel de la muñeca y a nivel del húmero distal.

Aplicando fuerzas en tracción hemos observado siempre roturas tipo I de Salter y Harris, 1963, donde la epífisis se separa completamente de la metáfisis observándose la anatomía de esta unión. La epífasis distal del fémur en el cordero, como también pasa en el hombre, presenta cuatro mamelones óseos, dos anteriores y dos posteriores, dos internos y dos externos, que penetran en cuatro cavidades a nivel de la metáfisis. Entre ambas superficies se dispone el cartílago de crecimiento que visto por delante ofrece una imagen en «V» que impide con esta forma la separación por solicitaciones de cizallamiento. En nuestro estudio se demuestra, como ya constatasen Weber y cols., 1980 y Rang, 1982, la función protectora sobre la fisis de las inserciones capsulares y los ligamentos de refuerzo mientras que como los autores señalados no hemos encontrado diferencias sexuales en el desplazamiento ni tampoco en la fuerza necesarias para producir la rotura fisaria, tal vez por no haber podido analizar la edad de los animales ya que las diferencias encontradas en la literatura se han encontraod cuando se hace un estudio comparativo de las diferentes edades.

\section{AGRADECIMIENTOS}

A la Fundación Ramón Areces por el patrocinio del proyecto de investigación gracias al cual ha sido elaborado el presente trabajo.

\section{BIBLIOGRAFÍA}

1. Aldegheri R, Trivella G, Lavini F. Epiphyseal distraction. Chondrodiastasis.Clin Orthop 1989, 241:117-27

2. Bright RW, Elmore, SM. Physical properties of epiphyseal plate cartilage. Surg Fonum, 1968; 19:463-65

3. Brighton CT Structure and function of the growth plate. Clin Orthop, 1978, 136: 22-32

4. Cañadell J, De Pablos J. Breaking bony bridges by physeal distraction. A new approach. Int Orthop, 1984; 9: 223-29

5. Cañadell J, De Pablos J. Correction of angular deformities by physeal distraction. Clin Orthop, 1992, 283: 98-105

6. Connolly JF, Huurman WW, Lippello L, Pankaj R. Epipyseal traction to correct acquired growth deformities. Clin Orthop, 1986, 202: 258-68

7. Crawford EJ; Jones $\mathrm{CB}$; Dewar ME; Aichroth PM. The force required to rupture the epiphysis in children undergoing epiphseal leg lengthening. XVII World Congress Sicot. Munich. August 16-21, 1985
8. De Bastini $G$, Aldegheri R, Renzi-Brivio L, Trivella G. Chondrodiastasis. Controlled symmetrical distraction of the epiphyseal plate. Limb lengthening in children. I Bone Joint Surg, 1986, 66B: 5506

9. De Bastiani $G$, Aldegheri R, Renzi-Brivio L, Trivella G. Limb lengthening by distraction of the epiphyseal plate. J Bone Joint Surg, 1986, 66B: $545-9$

10. De Pablos J, Cañadell J. Experimental physeal distraction in immature sheep. Clin Orthop, 1990, 250:73-80

11. De Pablos J, villas $\mathrm{C}$, Cañadell J. Bone lengthening by physeal distraction. An experimental study. Int Orthop, 1986, 10:163-70

12. Fishbane BM, Riley LH. Continous transphyseal traction: experimental observations. Clin Orthop, 1978, 136:120-4

13. Fjeld TO,Steen H. Limb lengthening by low rate epiphyseal distraction. An experimental study in the caprine tibia. J Orthop Res, 1988, 6:360-8

14. Harris WR. The endocrine basis for slipping of the upper femoral epiphysis. J Bone Joint Surg, 1950, 32-B: 5-11

15. Hert J. Regulation of the longitudinal growth of long bones. Experimental study onthe significance of mechanical factors. Lek Sbornik, suppl. 12, 1964; 114-23

16. Ilizarov GA, Soybelman LM. Some clinical and experimental data on the bloodless lengthening of the lower limbs. Exp Khir Anesth 1969, 4:27-32

17. Jani L Tierexperimentelle Studie über tibiaverlängerung durch Distraktionepiphseolyse. Z Orthop, 1973, 111:627-30

18. Jani L. Die Distraktionepiphseolyse: Tierexperimentelle Studie zum Problem der Beinverlängerung. Z. Orthop, 1975, 113: 189-98

19. Kuijpers-Jagtman AM, Bex JHM, Maltha JC, Daggers JG. Longitudinal growth of the rabbit femur after vascular and periosteal interference. Anat Anz, Jena 1988; 166:427-31

20. Letts RM, Meadows L. Epiphysiolysis as a method of limb lengthening. Clin Orthop, 1978, 133:230-7

21. Martinez JL, Mediero I, Ferrer M, Blanco E, Benito F. Ritmo de distracción epifisaria: estudio experimetnal. Rev Ortop Traum, 1992, 36IB:483-7

22. Monticelli G, Spinelli R: Distraction epiphysiolysis as a method of limb lengthening. I. experimental study. Clin Orthop, 1981, 154:254-61

23. Monticelli G, Spinelli R: Distraction epiphysiolysis as a method of limb lengthening. II. morphologic investigations. Clin Orthopo, 1981, 154:262-73

24. Monticelli G, Spinelli R. Distraction epiphysiolysis as a method of limb lengthening. III. clinical applications. Clin Orthop, 1981, 154:274-85

25. Mow VC, Holmes MH, LAI WM. Fluid transport and mechanical properties of articular cartilage. J Biomechanics, 1984; 17:377-94

26. Nakamura K, Matsushita $T$, Okazaki H, Nagano A, Kurokawa T. Attempted limb lengthening by physeal distraction. Clin Orthop, 1991, 267:306-11

27. Oliete V. Epifisiolisis traumática experimental. Tesis Doctoral. Universidad de Navarra, 1984

28. Pelker RR, Moen CT. Biomechanical and histological correlations in growth plate failure. Orthop Trans, 1983, 7, 274

29. Peltonen J, Alitalo I, Karaharju EO, Helio H. Distraction of the growth plate: experiments in pigs and sheep. Acta orthop scand, 1984, 55:35962

30. Pietravissa G. I distachi epifisari traumatici. Arch Putti di Chir. Org Mov, 1957, 8:153-92

31. Porter RW. The effect of tension across a growing epiphysis. J Bone Joint Surg, 1978, 10 B:252-6

32. Rang M. Childrens fractures. JB Lippincott Co, Philadelphia, Toronto, 1982

33. Ring $P A$. Experimental bone lengthening by epiphyseal distraction. $\mathrm{Br} \mathrm{J}$ Surg, 1958, 46: 169-73

34. Salter RB, Harris WR. Injuries involving the epiphyseal plate. J Bone Joint Surg, 1963, 45 A:587-622

35. Skak SV, Jensen TT, Poulsen TD, Stuurup J. Epidemiology of knee injuries in children. Acta Orthop scand, 1987; 58, 78-81

36. SledgeCB, Noble J. Experimental limb lengthening by epiphyseal distraction. Clin Orthop, 1978, 136:111-5

37. Steen $\mathrm{H}$, Fjeld TO, Ronningen $\mathrm{H}$, Langeland $\mathrm{N}$, Gjerdet N, Bjerkreim I. Limb lengthening by epiphyseal distraction. An experimental study in the caprine femur. J Orthop Res, 1987, 5:592-99

38. Tercedor J, Crespo V, Acosta F, Campos A, Fernandez E. Alargamiento tibial por distracción epifisaria proximal. Estudio experimental en conejos. Rev Ortop Traum, 1988, 32IB: 412-16

39. Weber $B G$, Brunner $C$, Freuler $F$. Treatment of fractures in children and adolescents. Springer Verlag, Berlin, Heidelberg, New York, 1980

40. Zavijalov PV, Plaskin JT. Distraction epiphysiolysis in lengthening of the lower ectremity inchildren. Khirurgiia, 1968, 44:121

41. Zavijalov PV, Plaskin JT. Elongation of crural bones in children using a method of distraction epiphysiolysis. Vestn Khir, 1967, 103:67. 